\title{
Astrometric results of observations of mutual occultations and eclipses of the Galilean satellites of Jupiter in 2003
}

\author{
N. V. Emelyanov ${ }^{1,2}$ and R. Gilbert ${ }^{2}$ \\ 1 Sternberg astronomical institute, 13 Universitetskij prospect, 119992 Moscow, Russia \\ e-mail: emelia@sai.msu.ru \\ 2 Institut de mécanique céleste et de calcul des éphémérides - Observatoire de Paris, UMR 8028 du CNRS, \\ 77 avenue Denfert-Rochereau, 75014 Paris, France
}

Received 5 January 2006 / Accepted 2 March 2006

ABSTRACT

\begin{abstract}
Context. The photometry of mutual occultations and the eclipses of natural planetary satellites can be used to infer very accurate astrometric data. This can be achieved by analyzing the light curves of the satellites observed during these mutual events.

Aims. The final goal of observations is to refine the models of motion for the natural satellites, and to develop a very accurate photometric model of mutual occultations and eclipses of satellites. This paper is focusing on the differences of topocentric or heliocentric coordinates of satellites pairs by analyzing the photometry of mutual occultations and the eclipses of natural satellites.

Methods. We propose the most accurate photometric model of mutual events to date based on all available data about the satellites, and have developed the corresponding method for extracting astrometric data. We analyze the errors of astrometric results obtained in terms of different scattering laws allowing for reflecting properties of the satellites surfaces. In addition we consider how to allow various previously neglected effects.

Results. We describe the results obtained by applying our method to observations of mutual occultations and eclipses of the Galilean satellites of Jupiter made in 2002-2003. We show that the coordinate errors due to irreducible systematic errors of photometry are about the same as the errors introduced by neglecting the above effects. We find the available maps of satellites surfaces to be unsuitable for deriving photometric event models.
\end{abstract}

Key words. occultations - eclipses - planets and satellites: general - astrometry

\section{Introduction}

Progress in studying the dynamics and in computating the ephemerides of natural planetary satellites depends on the accuracy of observations. Refining observational techniques and putting observational instruments beyond Earth's atmosphere evidently serve to improve the accuracy. However, such rare events as mutual occultations and eclipses of natural planetary satellites are also potentially very rich sources of high-precision astrometric data. Positional data can be derived from the photometry of satellites during such events. For a description of specific features of the mutual occultations and eclipses of satellites and for brief reviews of observations see Aksnes \& Franklin (1976), Aksnes et al. (1984), Arlot (1984), Franklin et al. (1991), Arlot et al. (1992), Vasundhara (1994), Emel'yanov (1995), Emelianov et al. (1999), Kaas et al. (1999), Emel'yanov (2000), Emelianov et al. (2000), Emel'yanov \& Vashkov'yak (2001), and Vasundhara et al. (2003).

Observations of both mutual occultations and eclipses of natural planetary satellites consist of measurements of the satellite flux during a single event. Extracting astrometric data from such photometry is an important but difficult task. The methods for solving this problem are now a new stage of development. Three groups of authors have been working extensively on the development of such methods. Decamps (1992) and Decamps et al. (1992) have thoroughly analyzed the photometric manifestations of the reflective properties of satellite surfaces during mutual occultations and eclipses. Vasundhara (1994) performed astrometric reduction of 1991 observations of mutual occultations and eclipses of the Galilean satellites. Aksnes \& Franklin (1976), Aksnes et al. (1984), Franklin et al. (1991), and Kaas et al. (1999) developed an approximate method for systematically infering and publishing the differences in the topocentric coordinates of the Galilean satellites based on the photometry of their mutual occultations and eclipses.

In our papers (Emel'yanov 1995, 1999, 2000) we suggested a new approach and developed an original method for inferring astrometric data from the photometry of mutual occultations and the eclipses of planetary satellites. For a description of the differences and its advantages of this method those proposed by other authors see Emel'yanov (1999), (2000), and (2003). The last of these gives a thorough description of the geometric model of the events considered and describes the algorithm for determining the satellite coordinates.

In this paper we consider the methods for considering various, previously neglected effects. We determine the individual contribution of each effect to the computed satellite coordinates, and analyze the following effects:

- wavelength-dependent solar limb darkening;

- various laws of light scattering by a rough surface; and

- variation of reflective properties over the satellite surface.

We use our new method to reduce the series of observations of mutual occultations and eclipses of the Gallilean satellites in 2002-2003 made at different observatories. 


\section{Photometric model of a mutual eclipse}

Consider now the process of how light is propagated from the Sun after its scattering by the surface of the satellite and until the light detector of the ground-based observer. Part of the solar disk seen from a point on the surface of the eclipsed satellite is covered by the eclipsing satellite.

Let us denote an infinitesimal element of the disk surface as $\mathrm{d} s_{0}$. The illumination of a point on the surface of the satellite produced by the element of the solar disk in spectral band $\mathrm{d} \Lambda \mathrm{de}$ pends on wavelength $\Lambda$ and angular distance $R_{\mathrm{c}}$ of this element from the disk center. This illumination is equal to

$I\left(\Lambda, R_{\mathrm{c}}\right) \mathrm{d} s_{0} \mathrm{~d} \Lambda$,

where function $I\left(\Lambda, R_{\mathrm{c}}\right)$ describes solar limb darkening. This formula allows for the well-known observational fact of the dependence of limb darkening on the wavelength.

The light from the given solar-disk element that illuminates the satellite is scattered by area $\mathrm{d} s_{\mathrm{p}}$ of the satellite surface toward the observer. The contribution of this light to the flux incident onto the detector is determined by the scattering properties of the element of the satellite surface and is equal to

$F(\varphi, \lambda, i, e, \alpha, \Lambda) I\left(\Lambda, R_{\mathrm{c}}\right) \mathrm{d} s_{0} \mathrm{~d} \Lambda \mathrm{d} s_{\mathrm{p}}$,

where function $F(\varphi, \lambda, i, e, \alpha, \Lambda)$ depends on the scattering properties of the satellite surface at the point with satellitographic coordinates $\varphi, \lambda$. It also depends on the angle of incidence, $i$, angle of reflection $e$, and phase angle $\alpha$ between the the rays incident onto and reflected from the surface, and on wavelength $\Lambda$.

When falling into the observer's detector, light produces an output signal equal to

$k_{\mathrm{a}} \Phi(\Lambda) F(\varphi, \lambda, i, e, \alpha, \Lambda) I\left(\Lambda, R_{\mathrm{c}}\right) \mathrm{d} s_{0} \mathrm{~d} \Lambda \mathrm{d} s_{\mathrm{p}}$,

where $\Phi(\Lambda)$ is the spectral sensitivity curve of the detector plus filter, and $k_{\mathrm{a}}$ is air-mass transparency.

The response of the detector to the flux from the eclipsed satellite can be computed by integrating (1) over the unocculted part of the solar disk $s_{0}$, over the part of the satellite surface $s_{\mathrm{p}}$ that is illuminated by the unocculted sun and observable from the Earth, and over detector sensitivity band $\Lambda_{1}-\Lambda_{2}$. Here we view $s_{0}$ and $s_{\mathrm{p}}$ not only as areas but also as certain parts of the solar disk and satellite surface, respectively, with known properties.

The detector is illuminated by the sky background and the light scattered by the parts of the instrument, in addition to the satellite light. Moreover, the photometric count also includes the signal produced by the detector itself. Hereafter we refer to the part of the photometric count that is not due to the satellite flux as the background, and denote it as $P$. During a mutual eclipse of satellites, the unocculted part of the solar disk varies to produce dependence $s_{0}(t)$ on time $t$.

The resulting simulated time-dependent photometric count $E(t)$ can be determined from the following formulas

$G^{(\mathrm{p})}(t)=\int_{s_{\mathrm{p}}} \int_{\Lambda_{1}}^{\Lambda_{2}} k_{\mathrm{a}} \Phi(\Lambda) F(\varphi, \lambda, i, e, \alpha, \Lambda) \int_{s_{0}(t)} I\left(\Lambda, R_{\mathrm{c}}\right) \mathrm{d} s_{0} \mathrm{~d} \Lambda \mathrm{d} s_{\mathrm{p}},(2)$

$E(t)=G^{(p)}(t)+P$.

Dependence $s_{0}(t)$ is determined by the relative positions of the satellites and can be modeled by the theory of their motion.
In relations (2), (3) we assume that the time variation of the photometric count is due to dependence $s_{0}(t)$ exclusively and neglect any explicit time dependence of other parameters. This assumption is justified by the short duration of the events considered. In the case of the Galilean satellites the durations of most of the mutual eclipses do not exceed 20 min. Let us, however, assess the errors that may be introduced by such an assumption. The part $s_{\mathrm{p}}$ of the satellite surface that is illuminated and can be observed from the Earth varies with time due to the rotation of the satellite during the mutual eclipse. This variation only shows up in the photometric count if the surface is substantially irregular and if event is very long. Air-mass transparency, $k_{\mathrm{a}}$, may vary because of the variation in the zenith angle of the satellite and because of eventual cloud movement. The first effect may only show up in very long events. Variations in background $P$ may be due mostly to the variation in the angle between the telescope axis and the direction toward the planet whose light is scattered by parts of the instrument. Noise can be partly eliminated using special methods of photometric reduction. In extreme cases, parameters $k_{\mathrm{a}}$ and $P$ can be fitted by linear functions of time with the coefficients determined from the observations to be reduced.

For a more convenient derivation of astrometric data from the photometry of the mutual eclipses of satellites, we transform relation (3) to the following form:

$E(t)=K S(t)+P$,

where

$S(t)=\frac{G^{(\mathrm{p})}(t)}{G^{(\mathrm{p})}\left(t_{1}\right)}, \quad K=G^{(\mathrm{p})}\left(t_{1}\right)$,

and $t_{1}$ denotes the instant in time immediately before the start of the event. In view of the above assumption about the constancy of parameters, $S(t)=1$ at all time instants before and after the event, and $K$ and $P$ always remain constant.

We consider the problem of finding and computing the functions that appear in the integrand in formula (2) in the next sections of this paper.

\section{Photometric model of a mutual occultation}

In the case of a mutual occultation of two satellites the points of their surfaces are always illuminated by the entire solar disk. The amount of light incident onto an infinitesimal element $\mathrm{d} s_{\mathrm{p}}$ of the surface of the occulted satellite within spectral band $\mathrm{d} \Lambda$ is equal to

\section{$J(\Lambda) \mathrm{d} \Lambda \mathrm{d} s_{\mathrm{p}}$,}

where function $J(\Lambda)$ describes the wavelength dependence of solar radiation.

During mutual occultations the images of two satellites are indistinguishable, and the measurements always record the total flux. Let us denote the element of the surface of the occulting satellite by $d s_{\mathrm{a}}$. The amount of light received by this element is then determined by the following formula

$J(\Lambda) \mathrm{d} \Lambda \mathrm{d} s_{\mathrm{a}}$.

Solar light incident onto the satellite is scattered by each area of the satellite toward the observer. The output signal of the detector corresponding to the flux from the occulted satellite is equal to

$G^{(\mathrm{p})}(t)=\int_{s_{\mathrm{p}}(t)} \int_{\Lambda_{1}}^{\Lambda_{2}} k_{\mathrm{a}} \Phi(\Lambda) F_{\mathrm{p}}(\varphi, \lambda, i, e, \alpha, \Lambda) J(\Lambda) \mathrm{d} \Lambda \mathrm{d} s_{\mathrm{p}}$ 
and the output signal corresponding to the occulting satellite is given by the following formula

$G^{(\mathrm{a})}=\int_{s_{\mathrm{a}}} \int_{\Lambda_{1}}^{\Lambda_{2}} k_{\mathrm{a}} \Phi(\Lambda) F_{\mathrm{a}}(\varphi, \lambda, i, e, \alpha, \Lambda) J(\Lambda) \mathrm{d} \Lambda \mathrm{d} s_{\mathrm{a}}$.

Here $s_{\mathrm{p}}(t)$ denotes the unocculted part of the surface of the occulted satellite that is illuminated by the Sun and visible from the Earth. This part of the surface varies with time due to the mutual motion of the apparent disks of the satellites, which is determined by the theory of their motion. Here $s_{\mathrm{a}}$ denotes the part of the occulting satellite that is illuminated by the Sun and is visible from the Earth. We assume that this part is constant because of the short duration of the event. Functions $F_{\mathrm{p}}(\varphi, \lambda, i, e, \alpha, \Lambda)$ and $F_{\mathrm{a}}(\varphi, \lambda, i, e, \alpha, \Lambda)$ are similar to function $F(\varphi, \lambda, i, e, \alpha, \Lambda)$ in the case of mutual eclipse, with the difference that the former and the latter refer to the occulted and occulting satellites, respectively. These functions differ, because natural planetary satellites differ in their reflective properties.

As a result, the total reaction $E$ of the detector can be determined by the following relation

$E(t)=G^{(\mathrm{a})}+G^{(\mathrm{p})}(t)+P$,

where $P$ is the combined effect of parasite signals considered in the previous section of the paper.

As for the case of mutual eclipse, we transform relation (8) to the following form:

$E(t)=K S(t)+P$

where

$S(t)=\frac{G^{(\mathrm{a})}+G^{(\mathrm{p})}(t)}{G^{(\mathrm{a})}+G^{(\mathrm{p})}\left(t_{1}\right)}, \quad K=G^{(\mathrm{a})}+G^{(\mathrm{p})}\left(t_{1}\right)$,

and $t_{1}$ denotes the time instant immediately preceding the start of the event. In view of the above assumption about the constancy of the parameters, $S(t)=1$ at all time instants before the start and after the end of the event, and $K$ and $P$ remain constant all the time.

We consider the functions that appear in the integrands in formulas (6) and (7) in the next sections of this paper. Note that a formula that is similar to (10) can be used in the cases of mutual eclipses where the total flux from the occulting and occulted satellites is measured. One must only use relation (2) instead of formula (6) to compute $G^{(\mathrm{p})}(t)$.

\section{Extraction of astrometric data from photometry of mutual occultations and eclipses of satellites}

Consider now the method for inferring positional and astrometric data from the measurements of satellite fluxes during their mutual occultations and eclipses. One of us has been systematically developing such a method for reduction of photometry in a number of papers (Emelianov et al. 1999, 2000; Emel'yanov $2000,2003)$. According to this method, function $S(t)$ is to be written in the form $S(X(t), Y(t))$, where $X(t)$ and $Y(t)$ are the projections of the differences of planetocentric Cartesian coordinates of the two satellites onto the plane of the event. In the case of mutual occultations, this plane coincides with the plane passing through the occulted satellite perpendicular to the line of sight of the observer. In the case of a mutual eclipse, the plane of the event passes through the eclipsed satellite perpendicular to the line connecting the satellite with the center of the Sun. The coordinate origin is placed at the center of the passive (occulted or eclipsed) satellite. The occulting or eclipsing satellite is referred to as the active satellite.

Given appropriate theories of the motion of planets and satellites, one can compute the theoretical values of functions $X(t), Y(t)$, i.e., $X_{\mathrm{th}}\left(t_{i}\right), Y_{\mathrm{th}}\left(t_{i}\right)$ for the time $t_{i} \quad(i=1,2, \ldots, m)$ of each photometric measurement. Here $m$ is the number of photometric counts during a single event. The real values of $X(t)$ and $Y(t)$ differ from $X_{\mathrm{th}}\left(t_{i}\right)$ and $Y_{\mathrm{th}}\left(t_{i}\right)$ by corrections $D_{x}, D_{y}$; i.e.,

$X(t)=X_{\mathrm{th}}\left(t_{i}\right)+D_{x}, \quad Y(t)=Y_{\mathrm{th}}\left(t_{i}\right)+D_{y}$.

The underlying assumption of the method is that corrections $D_{x}$ and $D_{y}$ remain constant during the observed event. According to our estimates, the error introduced by this assumption for Galilean satellites does not exceed $3.7 \mathrm{~km}$ in the satellite coordinates. To achieve better accuracy, corrections $D_{x}$ and $D_{y}$ should be fitted by linear functions of time. In this case, the error should not exceed $0.025 \mathrm{~km}$. The results of reducing modern observations show that with the actual errors of the satellite coordinates the corrections $D_{x}$ and $D_{y}$ can be considered to be constant.

Our proposed method consists of solving conditional equations

$$
\begin{array}{r}
E_{i}=K S\left(X_{\mathrm{th}}\left(t_{i}\right)+D_{x}, Y_{\mathrm{th}}\left(t_{i}\right)+D_{y}\right)+P \\
(i=1,2, \ldots, m)
\end{array}
$$

for constants $D_{x}, D_{y}, K$, and $P$. Here $E_{i}$ is the photometric count made at time $t_{i}$. We linearize conditional equations with respect to parameters $D_{x}, D_{y}$ and then solve them using the least-square method.

The result of reducing the photometric observations of a single event can be taken to consist of the following values:

$X=X_{\mathrm{th}}\left(t^{*}\right)+D_{x}, \quad Y=Y_{\mathrm{th}}\left(t^{*}\right)+D_{y}$,

where $t^{*}$ is an arbitrary time instant inside the event interval. Let us assume that it is the time instant when $\sqrt{X^{2}+Y^{2}}$ takes its minimum value; i.e., $t^{*}$ is the time of the closest apparent approach of the satellites. Given the topocentric distances of the active and passive satellites, one can compute the corresponding projections $X^{\prime \prime}$ and $Y^{\prime \prime}$ of the angular separation between the satellites onto the celestial parallel and meridian, respectively. It goes without saying that the topocenter is taken to coincide with the center of the Sun in the case of a mutual eclipse. In this case the time instants for which the positions of the satellites are determined do not coincide with the time of observation. The relations between these time instants can be found in (Emel'yanov 2000).

If the same event was observed at two observatories, the time instants $t^{*}$ may differ because of observational errors, and the differences between $X^{\prime \prime}$ and $Y^{\prime \prime}$ obtained at two observatories cannot be viewed as bias indicators. However, the corrections $D_{x}$ and $D_{y}$ remain constant during the event and characterize the discrepancy between the theory and observations. These values obtained from observations made at different observatories can be compared to each other. The resulting discrepancies are due to observational biases. The internal accuracy of photometry as estimated from the least-square method is characterized by the errors $\sigma_{x}$ and $\sigma_{y}$ of the inferred parameters $X^{\prime \prime}$ and $Y^{\prime \prime}$.

Our analysis leads us to the following two important conclusions. First, joint least-square determination of four parameters $D_{x}, D_{y}, K$, and $P$ may result in highly correlated parameter errors, and the differences between the inferred parameters 
and their real values may exceed the random errors substantially. That is why it is better not to treat parameter $P$ (background) as an unknown, but eliminate the effect of the background on the result of measurement beforehand and set $P=0$. Second, if we set $P=0$ while incorrectly eliminating background so that this parameter actually differs from zero, an unaccounted systematic error appears in parameter $D_{y}$.

Another source of possible error is that any observed light curve of the satellites has two solutions for the corresponding coordinate differences of apparent coordinates of satellites. The occulting satellite may pass above or below the occulted satellite at the same distance from the latter producing the same dips in the total flux. This property is obeyed exactly if the apparent relative motion of the occulting satellite is constant and the disks of the satellites are absolutely uniform. Of the two solutions we may choose the one that agrees better with the available theory of motion. However, this approach may result in selecting the wrong solution if the theory is not accurate enough. In the cases where the apparent disk of one of the satellites overlaps fully with the disk of the other satellite, the solution becomes uncertain in one of the relative coordinates. In these cases we can only give the interval of possible values for the corresponding coordinate. Photometric errors make this uncertainty more likely. The above effects also occur in the case of the mutual eclipses of satellites.

Note that the methods developed by Vasundhara (1994), Kaas et al. (1999), and Vasundhara et al. (2003) all face similar problems.

\section{Properties of satellites and simplifications adopted in the photometric model}

Let us now consider the integrands that appear in relations (2), (6), and (7). The model of photometric measurements includes only the ratio of the integrals of these functions computed over various domains: the entire solar disk and its part in the case of an eclipse, or the entire surface of the occulted satellite and its unocculted part in the case of a mutual occultations. The functions can therefore be specified up to an arbitrary constant factor, i.e., we can use just their relative values.

Spectral sensitivity functions $\Phi(\Lambda)$ of the detector plus filter can be obtained from laboratory tests of the instrument or adopted from the documentation of the instruments employed. These data must accompany the results of photometric observations of the satellites. Functions $I\left(\Lambda, R_{\mathrm{c}}\right)$ and $J(\Lambda)$ are obtained from solar studies. Tables of these functions can be adopted from Makarova et al. (1998). Function $F(\varphi, \lambda, i, e, \alpha, \Lambda)$ describes the scattering properties of each point on the satellite surface. The intensity of scattered light depends on the mutual orientation of the incident light ray, reflected light ray, and the normal to the surface. This dependence may differ at different points of the surface and at different wavelengths. Such detailed data about the properties of the surfaces of the Galilean satellites are not likely to become available at present or in the very near future. Therefore function $F(\varphi, \lambda, i, e, \alpha, \Lambda)$ should involve inevitable simplifications.

The task is very difficult and therefore, in practice, function values averaged over some spectral band are used instead of wavelength dependence. Let us denote the fixed spectral band as $N$. If the satellite is observed in the spectral band $N$, we can assume that

$F(\varphi, \lambda, i, e, \alpha, \Lambda)=A_{N}(\varphi, \lambda) f_{N}(i, e, \alpha) \cos e$, where $A_{N}(\varphi, \lambda)$ can be determined from the maps of the satellite surface made in the spectral band $N$, and $f_{N}(i, e, \alpha)$ is the scattering law with the parameters determined for this band.

We used two of the wide variety of scattering laws considered in planetology: the Lommel-Seeliger law and Hapke scattering law. Hapke $(1981,1984)$ analyzed what is so far the most detailed and general law of scattering by the surface of a celestial body. His scattering function $f_{N}(i, e, \alpha)$ depends, in addition to the arguments mentioned above, on five more parameters, which are constant for the given satellite and spectral band.

We could only find two complete sets of published Hapke parameters for the Galilean satellites. The first paper (McEwen et al. 1988) gives the Hapke parameters for the rough surface of the Io satellite. The authors of the second paper (Domingue \& Verbiscer 1997) refined the Hapke function for rough surfaces. The scattering function includes a number of empiric relations.

To allow for the nonuniformity of the satellite surface in the photometric model of mutual occultations and eclipses, we tried to use the maps of the Galilean satellites of Jupiter. We downloaded these maps in JPG graphical format from the Internet in October 2004. We downloaded the map of Io from http://jupiter . berkeley.edu/maps/newv2mos_gr.jpg, and those of the other three satellites from the site

http: //astrogeology .usgs.gov at the following addresses: /Projects/JupiterSatellites/europa/europa_simp_8km.jpg, /Projects/JupiterSatellites/gany/gany_simp_8km.jpg,

/Projects/JupiterSatellites/callisto/callisto_simp_8km .jpg, for Europa, Ganymede, and Callisto, respectively. For the satellite maps to be usable in our computational program for the reduction of observations, the image files were converted into ASCII files where each pixel was replaced by an integer representing its intensity. The intensity that we have adopted for the pixels belonging to uncharted regions was the median value of all the pixels of the charted regions.

The brightness of each point on the disk can be computed using the appropriate scattering law and the satellite map. It is thus possible to compute the mean brightness of the disk as a function of the angle of rotation of the satellite.

In the cases where the satellite rotation is synchronous with its orbital motion, angle of rotation $\theta$ is determined as the planetocentric angle between the direction toward the satellite and the projection of the line of sight of the ground-based observer onto the orbital plane. In this case $\theta=0$ and $\theta=90^{\circ}$ in the upper conjunction and Eastern elongation, respectively.

On the other hand, this function can be determined from the ground-based photometry of a particular satellite viewed as a point light source. We know only one paper (Morrison \& Morrison 1977) whose authors report the dependence of the magnitudes of Galilean satellites on angle of rotation obtained from single-band $(V)$ ground-based photometry.

The data on the dependence of the satellite brightness on the angle of rotation reported in the two sources mentioned above were compared to each other to test the reliability of the data. To this end, we modeled the integrated brightness of the satellite at different angles of rotation based on the surface maps mentioned above and assuming the Hapke scattering law with the above-mentioned Hapke parameters for the Galilean satellites. Figures 1-4 show the results of map-based modeling and the results of ground-based photometry. The ordinate axes in the plots give the flux $S$ from the satellite expressed in fractions of its mean value averaged over all angles of rotation $\theta$.

As is evident from the plots, the modeled and observed dependences for Io and Europa differ insignificantly. However, the differences for Ganymede and Callisto are greater. 


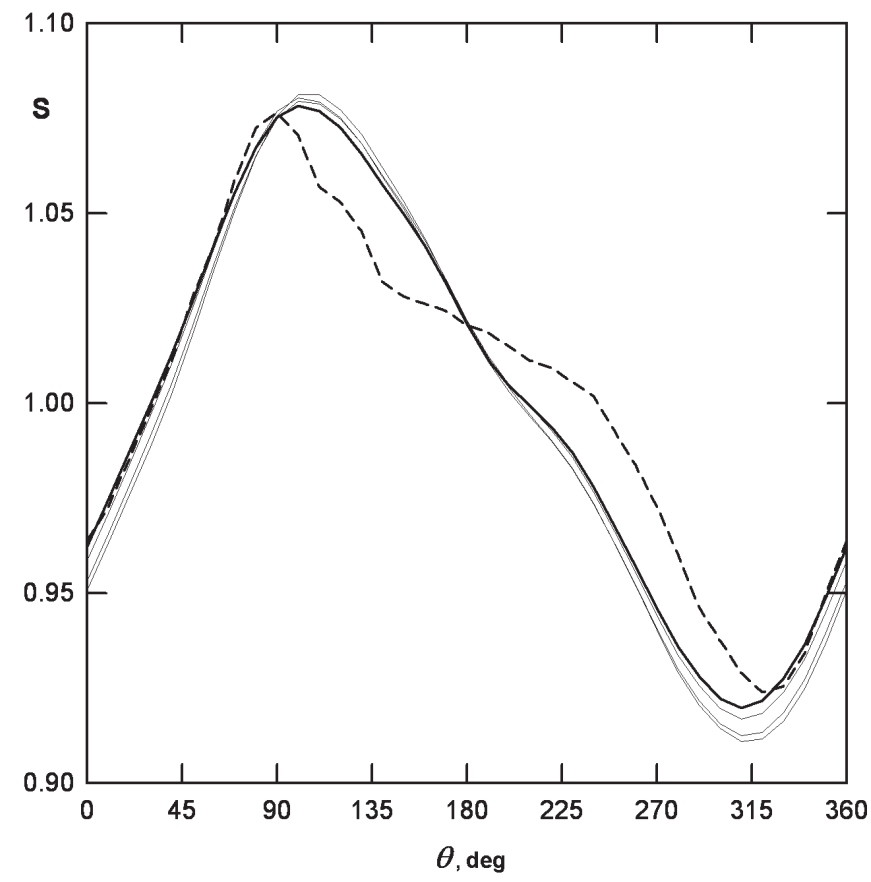

Fig. 1. The integrated brightness of the satellite Io at different angles of rotation $\theta$ based on the surface maps (bold line - the phase angle $\alpha=0$, thin lines - the phase angle $\alpha=4,8,12$ degrees) and the results of ground-based photometry (dashed line).

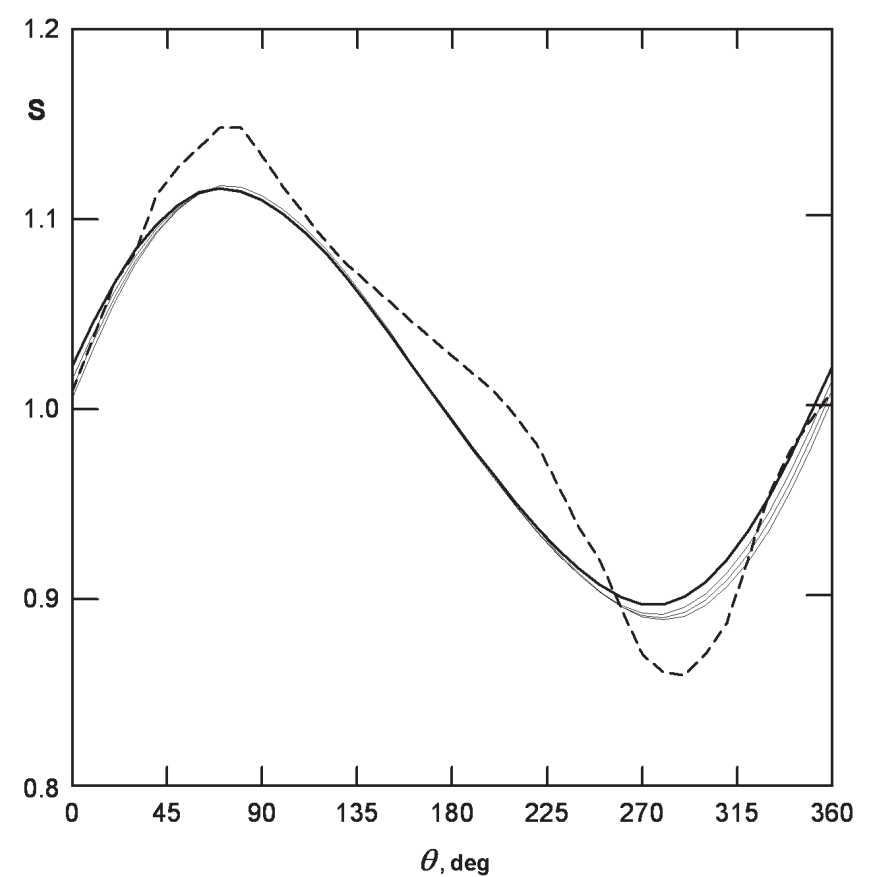

Fig. 2. The integrated brightness of the satellite Europa at different angles of rotation $\theta$ based on the surface maps (bold line - the phase angle $\alpha=0$, thin lines - the phase angle $\alpha=4,8,12$ degrees) and the results of ground-based photometry (dashed line).

A brief verification of the integrated brightness curve can be made by rule of thumb. On the map of Callisto centered at longitude 0 degrees, the left part is much brighter than the right. Therefore, using a 180 degree wide "window" that moves from the center of the picture toward west and then loops back on the right side toward the center, we can estimate that the total

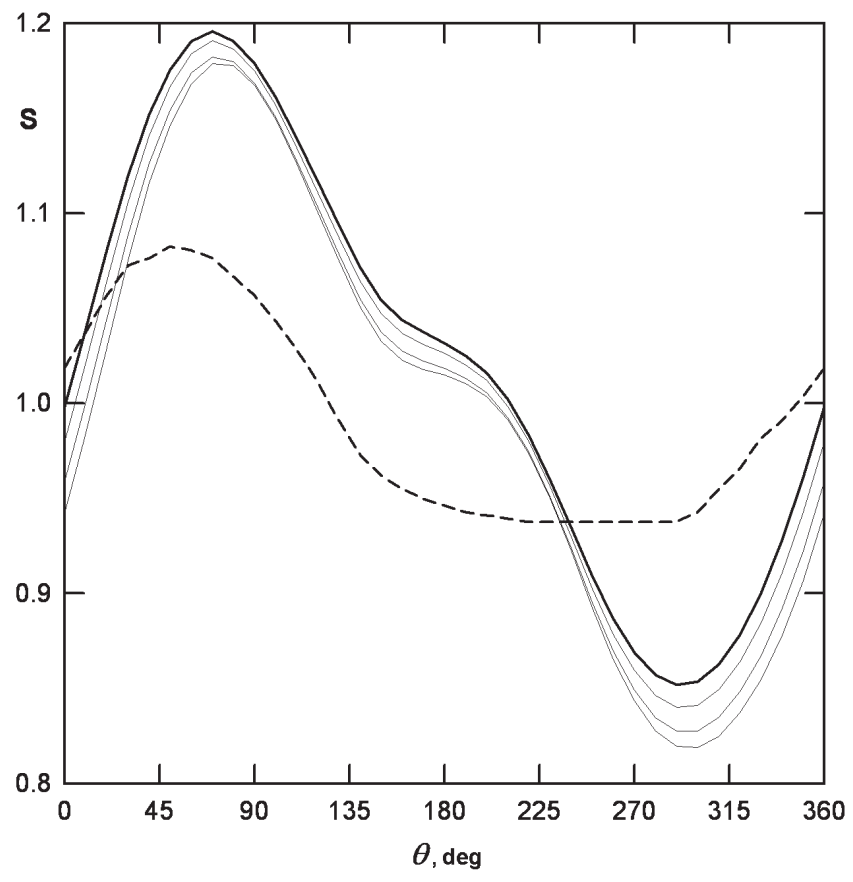

Fig. 3. The integrated brightness of the satellite Ganymede at different angles of rotation $\theta$ based on the surface maps (bold line - the phase angle $\alpha=0$, thin lines - the phase angle $\alpha=4,8,12$ degrees) and the results of ground-based photometry (dashed line).

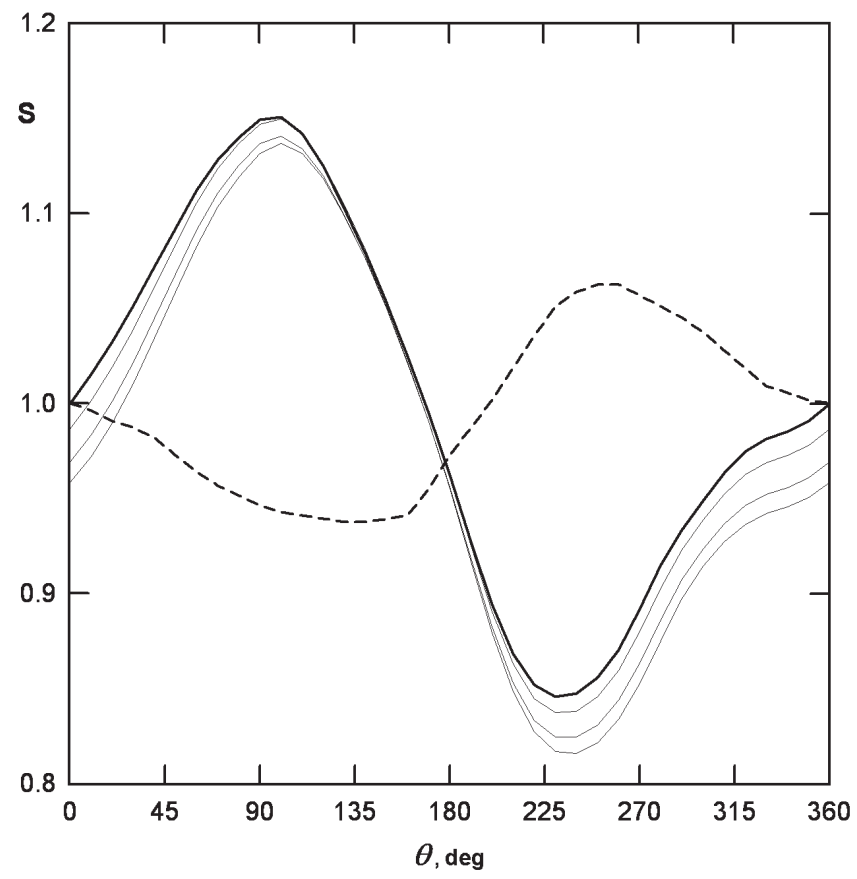

Fig. 4. The integrated brightness of the satellite Callisto at different angles of rotation $\theta$ based on the surface maps (bold line - the phase angle $\alpha=0$, thin lines - the phase angle $\alpha=4,8,12$ degrees) and the results of ground-based photometry (dashed line).

brightness through the window will first increase and then will decrease when looping back. This observation concords with the results of our brightness curve, in particular, for Callisto.

These maps are actually intended for other purposes, and they describe the brightness distribution across the surface only approximately. Despite the circumstances mentioned above we 
have no other alternative for allowing for the nonuniformity of the satellite surfaces but to use the available maps.

Let us now consider the contribution of the solar-limb darkening to the astrometric results obtained from the photometry of mutual occultations and eclipses of planetary satellites. We estimated this contribution by simulating photometric measurements under close-to-real conditions. We used the eclipse of Io by Ganymede on September 14, 1997 as an example, and computed simulated observations with the allowance for the wavelength-dependent solar-limb darkening. We then used these simulated observations to refine the projections of satellite coordinates onto the event plane using a method that neglects limb darkening. The error in the difference of projected coordinates was equal to 22 and $60 \mathrm{~km}$ along the trajectory of the occulting satellite and in the perpendicular direction, respectively.

We also analyzed how the results depend on the allowance for the spectral dependence of solar limb darkening. We simulated observations in the example considered above with an allowance for the color dependence of limb darkening and refined the satellite coordinate differences taking monochromatic limb darkening for the green wavelength into account. The errors were equal to 4 and $10 \mathrm{~km}$ in the two directions mentioned above.

Our analysis also leads us to the conclusion that, in the case of mutual occultations of planetary satellites, dependence $S(t)$ is determined to a considerable degree by the ratio of the mean albedos of the two satellites. Small errors in the adopted albedos translate into errors in the inferred coordinate differences of the satellites.

\section{Results of the observations of mutual occultations and eclipses of the Galilean satellites in 2003}

The results of photometric observations of the Gallilean satellites during their mutual occultations and eclipses in 2003 performed at various observatories worldwide were compiled at the Institut de mécanique céleste et de calcul des éphémérides (IMCCE), France, and made available to the authors of this paper. The entire database includes 290 light curves of satellites obtained at 36 observatories in the world. A total of 1 to 14 events were observed at each observatory. Some of the events were observed simultaneously at 10 observatories. These data may be incomplete, because reduction of observations is still in progress. Also the reductions of the observations were obtained directly from raw data provided by IMCCE. Thus, no light curves had been pre-processed or altered by anyone before our treatment.

We plan to reduce all the available data in the future, and at the current stage we have restricted our analysis to the events that were observed simultaneously at more than one observatory. A comparison of the results makes it possible to identify and estimate the systematic errors of observations. Table 1 lists the observatories where the results of the observations of the events considered here were obtained.

We inferred the astrometric results from the photometry of satellites during their mutual occultations and eclipses using the method described in detail by Emel'yanov (2000, 2003). We computed the theortical values $X_{\text {th }}\left(t_{i}\right)$ and $Y_{\text {th }}\left(t_{i}\right)$ and corrections $D_{x}^{\prime \prime}$ and $D_{y}^{\prime \prime}$ using the theory of Galilean satellite motion developed by Lainey et al. (2005). Compared to our previous applications of this method, the advantages of this work consist in the use of various scattering laws and in the allowance for the nonuniformity of the satellite surface. For some of the events,

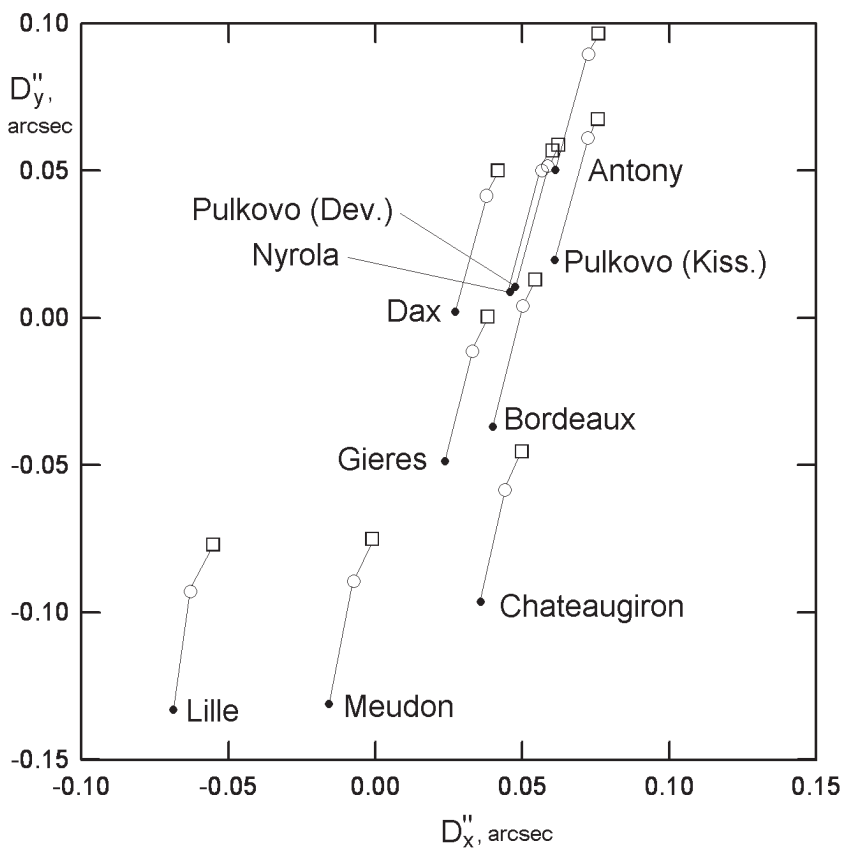

Fig. 5. The astrometric results from the photometry during the eclipse of Callisto by Ganymede on March 15, 2003. The values of $D_{x}^{\prime \prime}$ and $D_{y}^{\prime \prime}$ obtained from observations made at different observatories are shown. Dots - with the complete allowance for all effects including the Hapke scattering law and the nonuniformity of the satellite surface accounted for in accordance with the available maps; squares - the same variant but assuming that the surface is uniform; circles - adopting the Lommel-Seeliger law under the assumption of uniform surface.

we performed three variants of reduction: (1) with the complete allowance for all effects considered above including the Hapke scattering law and the nonuniformity of the satellite surface accounted for in accordance with the available maps; (2) the same as variant (1) but assuming that the surface is uniform; and (3) adopting the Lommel-Seeliger law under the assumption of a uniform surface.

In the reported results mutual occultations and eclipses are designated as $A o P$ and $A e P$, respectively, where $A$ is the number of the active (occulting or eclipsing) satellite and $P$ the number of the passive (occulted or eclipsed) satellite.

To compare the results obtained in different variants of reduction and to confront the results of observations made at different observatories, we analyzed the quantities $D_{x}^{\prime \prime}$ and $D_{y}^{\prime \prime}$, which characterize the discrepancy between the theory and observations. Figures 5-8 show the results of reduction. These results lead us to conclude that the differences between the results of observations obtained at different observatories have the same order of magnitude as the differences between the results obtained in different variants of reduction.

Table 2 lists the astrometric data inferred with variant (1) of reduction from the photometry of a number of mutual occultations and eclipses of the Galilean satellites. The data to be used for further analysis is listed in the first three columns of the table. The next two columns give the errors of the inferred $X^{\prime \prime}$ and $Y^{\prime \prime}$ estimated using the least-square method. The quantities $D_{x}^{\prime \prime}$ and $D_{y}^{\prime \prime}$ characterize the agreement between theory and observations. These quantities can be used to compare the results of observations made at different observatories. The column $S_{\text {min }}$ can be used to compare the decrease in satellite brightness measured at different observatories. The last column gives the conventional 


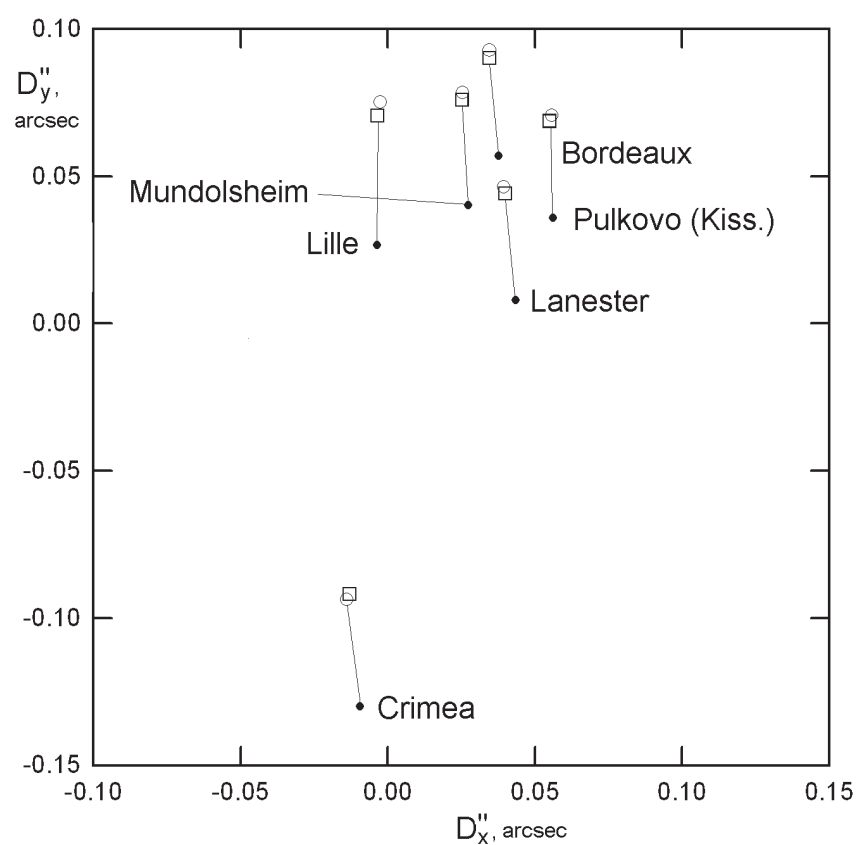

Fig. 6. The astrometric results from the photometry during the eclipse of Ganymede by Callisto on February 18, 2003. (See explanations to the Fig. 5).

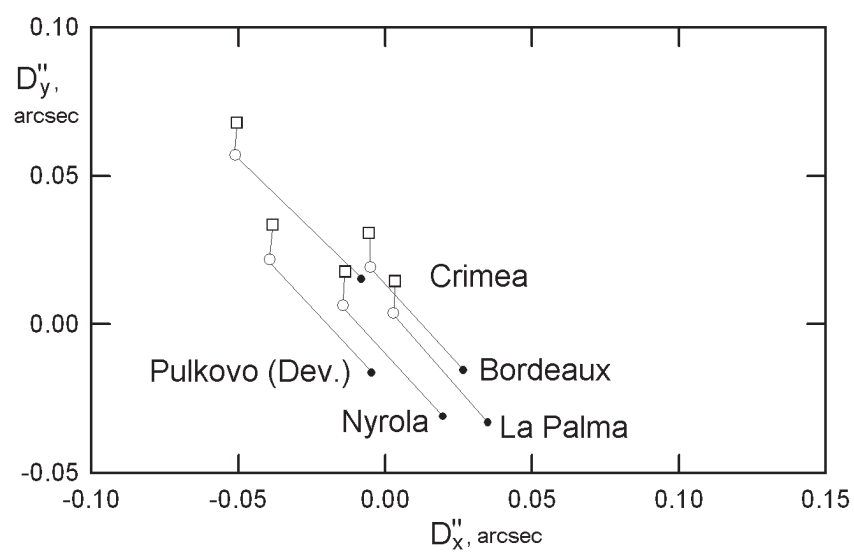

Fig. 7. The astrometric results from the photometry during the eclipse of Ganymede by Europa on March 25, 2003. (See explanations to the Fig. 5.)

designations of the observatories corresponding to those used in Table 1.

\section{Conclusions}

Our analysis and the results from reduction of a series of photometric observations of the Galilean satellites made during their mutual occultations and eclipses in 2003 lead us to the following conclusions.

- The published parameters of the Galilean satellites are evidently insufficient for the most refined Hapke scattering law. These parameters have been determined only for a single spectral band, whereas observations can be performed using different filters or in the "white" light, i.e., with no filters at all.

- The allowance for the nonuniformity of the reflective properties of the satellites based on published maps of the Galilean

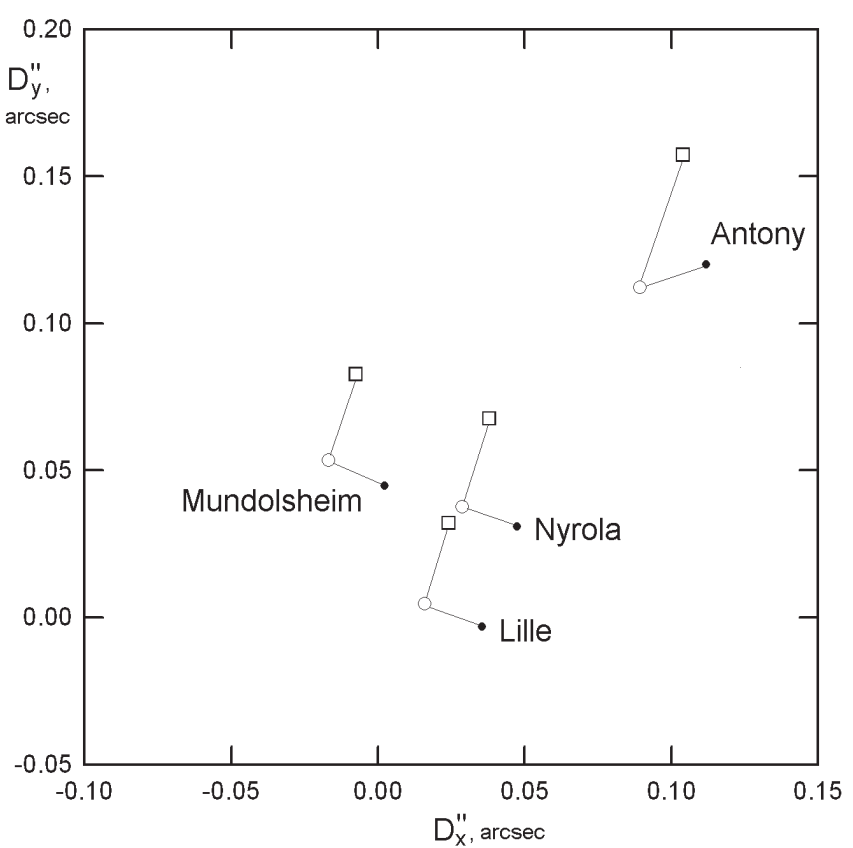

Fig. 8. The astrometric results from the photometry during the eclipse of Io by Callisto on February 19, 2003. (See explanations to the Fig. 5).

Table 1. List of observatories.

\begin{tabular}{cl}
\hline \hline $\begin{array}{c}N \\
\text { obs }\end{array}$ & $\begin{array}{l}\text { Name } \\
\text { of observatory }\end{array}$ \\
\hline B & Bordeaux - France \\
C & Catania - Italy \\
I & Gieres - France \\
J & Chateaugiron \\
K & Pulkovo (Kisseleva) - Russia \\
L & Lille - France \\
La & Lanester - France \\
N & Nyrola - Finland \\
O & Antony - France \\
T & Nauchny (Crimea) - Russia \\
U & Mundolsheim - France \\
X & Dax - France \\
Y & Pulkovo (Devyatkin) - Russia \\
\hline
\end{tabular}

satellites remains doubtful because of the discrepancy between these data and the ground-based photometry of the satellites made at different phase angles.

- The systematic errors of the astrometric results, as inferred from the observations that we estimated by comparing observations made at different observatories, have about the same magnitude as the effects of the allowance or non-allowance for various scattering laws and of the nonuniformity of the reflective properties of the satellite surfaces. The allowance for the above effects is of no use before the systematic errors of observations of the events considered are reduced considerably.

- The systematic errors of the photometry of satellites during their mutual occultations and eclipses are most likely due to not correctly allowing for the effect of background $(P)$ on photometric counts. 
Table 2. The astrometric data inferred from the photometry of a number of mutual occultations and eclipses by the Galilean satellites. The reduction was performed with the Hapke scattering law and the nonuniformity of the satellite surface. Special lines give the dates and types of the observed phenomena.

\begin{tabular}{|c|c|c|c|c|c|c|c|c|}
\hline $\begin{array}{c}t^{*}(T T) \\
\text { day }\end{array}$ & $\begin{array}{l}X^{\prime \prime} \\
\prime \prime\end{array}$ & $\begin{array}{l}Y^{\prime \prime} \\
\prime \prime\end{array}$ & $\begin{array}{c}\sigma_{x} \\
\prime \prime\end{array}$ & $\sigma_{y}$ & $\begin{array}{r}D_{x}^{\prime \prime} \\
\prime \prime\end{array}$ & $\begin{array}{r}D_{y}^{\prime \prime} \\
\prime \prime\end{array}$ & $S_{\min }$ & $\begin{array}{c}N \\
\text { obs }\end{array}$ \\
\hline \multicolumn{9}{|c|}{$2003.01 .06-2 \mathrm{e} 1$} \\
\hline 0.981827 & 0.0943 & 0.2938 & 0.001 & 0.001 & 0.005 & -0.022 & 0.50 & B \\
\hline 0.981780 & 0.0941 & 0.2929 & 0.009 & 0.008 & -0.003 & -0.021 & 0.50 & $\mathrm{C}$ \\
\hline 0.981774 & 0.1017 & 0.3168 & 0.002 & 0.002 & 0.004 & 0.003 & 0.53 & $\mathrm{~K}$ \\
\hline 0.981781 & 0.1041 & 0.3243 & 0.001 & 0.001 & 0.007 & 0.010 & 0.54 & $\mathrm{~N}$ \\
\hline 0.981818 & 0.1501 & 0.4675 & 0.006 & 0.004 & 0.059 & 0.152 & 0.72 & $\mathrm{Y}$ \\
\hline \multicolumn{9}{|c|}{$2003.02 .18-4 \mathrm{e} 3$} \\
\hline 0.867889 & -0.1880 & -0.5425 & 0.007 & 0.006 & 0.038 & 0.057 & 0.58 & B \\
\hline 0.867601 & -0.1238 & -0.3572 & 0.023 & 0.032 & 0.032 & 0.266 & 0.44 & $\mathrm{~J}$ \\
\hline 0.867983 & -0.1925 & -0.5555 & 0.009 & 0.007 & 0.056 & 0.036 & 0.59 & $\mathrm{~K}$ \\
\hline 0.867775 & -0.2017 & -0.5821 & 0.013 & 0.012 & -0.003 & 0.027 & 0.60 & $\mathrm{~L}$ \\
\hline 0.867972 & -0.2026 & -0.5845 & 0.045 & 0.036 & 0.043 & 0.008 & 0.61 & $\mathrm{La}$ \\
\hline 0.867954 & -0.2509 & -0.7240 & 0.002 & 0.001 & -0.009 & -0.130 & 0.71 & $\mathrm{~T}$ \\
\hline 0.867872 & -0.1943 & -0.5608 & 0.029 & 0.024 & 0.027 & 0.040 & 0.59 & $\mathrm{U}$ \\
\hline \multicolumn{9}{|c|}{$2003.02 .19-4 \mathrm{o} 1$} \\
\hline 0.855624 & -0.1192 & -0.3705 & 0.019 & 0.020 & 0.035 & -0.003 & 0.47 & $\mathrm{~L}$ \\
\hline 0.855623 & -0.1086 & -0.3375 & 0.004 & 0.005 & 0.047 & 0.031 & 0.45 & $\mathrm{~N}$ \\
\hline 0.855679 & -0.0760 & -0.2364 & 0.023 & 0.039 & 0.112 & 0.120 & 0.39 & $\mathrm{O}$ \\
\hline 0.855550 & -0.1083 & -0.3366 & 0.034 & 0.041 & 0.002 & 0.045 & 0.45 & $\mathrm{U}$ \\
\hline \multicolumn{9}{|c|}{$2003.03 .15-3 \mathrm{e} 4$} \\
\hline 0.927766 & 0.1064 & 0.2988 & 0.003 & 0.004 & 0.040 & -0.037 & 0.35 & B \\
\hline 0.927722 & 0.1009 & 0.2833 & 0.006 & 0.009 & 0.024 & -0.049 & 0.34 & I \\
\hline 0.927828 & 0.0872 & 0.2448 & 0.019 & 0.029 & 0.036 & -0.096 & 0.31 & $\mathrm{~J}$ \\
\hline 0.927769 & 0.1267 & 0.3557 & 0.003 & 0.004 & 0.061 & 0.020 & 0.39 & $\mathrm{~K}$ \\
\hline 0.927493 & 0.0638 & 0.1792 & 0.009 & 0.014 & -0.069 & -0.133 & 0.27 & $\mathrm{~L}$ \\
\hline 0.927685 & 0.0704 & 0.1977 & 0.005 & 0.008 & -0.015 & -0.131 & 0.28 & M \\
\hline 0.927727 & 0.1216 & 0.3412 & 0.003 & 0.003 & 0.046 & 0.009 & 0.38 & $\mathrm{~N}$ \\
\hline 0.927670 & 0.2062 & 0.5785 & 0.022 & 0.019 & 0.117 & 0.251 & 0.59 & $\mathrm{U}$ \\
\hline 0.927669 & 0.1173 & 0.3294 & 0.015 & 0.018 & 0.027 & 0.002 & 0.37 & $\mathrm{X}$ \\
\hline 0.927732 & 0.1223 & 0.3434 & 0.004 & 0.004 & 0.048 & 0.010 & 0.38 & $\mathrm{Y}$ \\
\hline \multicolumn{9}{|c|}{$2003.03 .18-2 \mathrm{e} 3$} \\
\hline 0.866879 & 0.0176 & 0.0496 & 0.008 & 0.020 & 0.028 & -0.197 & 0.61 & B \\
\hline 0.866777 & 0.1284 & 0.3631 & 0.009 & 0.010 & 0.104 & 0.129 & 0.68 & $\mathrm{C}$ \\
\hline 0.866852 & -0.0027 & -0.0076 & 0.016 & 0.000 & -0.001 & -0.251 & 0.61 & $\mathrm{H}$ \\
\hline 0.866908 & -0.0002 & -0.0007 & 0.033 & 0.000 & 0.021 & -0.251 & 0.61 & $\mathbf{J}$ \\
\hline 0.866826 & 0.0823 & 0.2330 & 0.011 & 0.019 & 0.075 & -0.007 & 0.64 & $\mathrm{~L}$ \\
\hline 0.866810 & 0.0758 & 0.2143 & 0.005 & 0.010 & 0.063 & -0.024 & 0.63 & $P$ \\
\hline 0.866720 & 0.1281 & 0.3625 & 0.012 & 0.015 & 0.084 & 0.135 & 0.68 & $\mathrm{U}$ \\
\hline \multicolumn{9}{|c|}{$2003.03 .24-1 \mathrm{e} 3$} \\
\hline 0.918669 & -0.2815 & -0.7874 & 0.007 & 0.003 & -0.008 & -0.022 & 0.88 & B \\
\hline 0.918976 & -0.2904 & -0.8127 & 0.021 & 0.011 & 0.045 & -0.069 & 0.89 & $\mathrm{H}$ \\
\hline 0.918996 & -0.2709 & -0.7578 & 0.014 & 0.007 & 0.069 & -0.016 & 0.86 & $\mathrm{~L}$ \\
\hline 0.918888 & -0.2961 & -0.8284 & 0.061 & 0.030 & 0.022 & -0.079 & 0.90 & $\mathrm{U}$ \\
\hline 0.918780 & -0.2901 & -0.8116 & 0.027 & 0.013 & 0.005 & -0.054 & 0.89 & $\mathrm{Y}$ \\
\hline \multicolumn{9}{|c|}{$2003.03 .25-1 \mathrm{e} 3$} \\
\hline 0.840112 & -0.2768 & -0.7710 & 0.009 & 0.004 & 0.010 & 0.024 & 0.87 & $\mathrm{~B}$ \\
\hline 0.840375 & -0.2836 & -0.7902 & 0.047 & 0.022 & 0.073 & -0.020 & 0.88 & $\mathrm{C}$ \\
\hline 0.839906 & -0.2618 & -0.7292 & 0.026 & 0.013 & -0.028 & -0.085 & 0.84 & $\mathrm{H}$ \\
\hline 0.840088 & -0.2963 & -0.8251 & 0.046 & 0.021 & -0.015 & -0.028 & 0.90 & $\mathrm{~L}$ \\
\hline 0.840075 & -0.3172 & -0.8835 & 0.016 & 0.007 & -0.040 & -0.085 & 0.93 & $\mathrm{~N}$ \\
\hline 0.840059 & -0.3121 & -0.8691 & 0.006 & 0.002 & -0.038 & -0.069 & 0.92 & $\mathrm{~T}$ \\
\hline 0.840110 & -0.3169 & -0.8827 & 0.022 & 0.009 & -0.030 & -0.087 & 0.93 & $\mathrm{Y}$ \\
\hline \multicolumn{9}{|c|}{$2003.03 .25-2 \mathrm{o} 3$} \\
\hline 0.867566 & -0.2346 & -0.7761 & 0.019 & 0.010 & 0.027 & -0.015 & 0.90 & B \\
\hline 0.867559 & -0.2399 & -0.7933 & 0.009 & 0.005 & 0.020 & -0.031 & 0.91 & $\mathrm{~N}$ \\
\hline 0.867599 & -0.2386 & -0.7890 & 0.021 & 0.011 & 0.035 & -0.033 & 0.91 & $\mathrm{P}$ \\
\hline 0.867457 & -0.2293 & -0.7582 & 0.004 & 0.002 & -0.008 & 0.015 & 0.90 & $\mathrm{~T}$ \\
\hline 0.867490 & -0.2279 & -0.7866 & 0.021 & 0.011 & -0.005 & -0.016 & 0.91 & $\mathrm{Y}$ \\
\hline \multicolumn{9}{|c|}{$2003.03 .26-2 \mathrm{e} 1$} \\
\hline 0.862302 & -0.2035 & -0.5753 & 0.023 & 0.011 & -0.016 & 0.015 & 0.85 & $\mathrm{C}$ \\
\hline 0.862340 & -0.2214 & -0.6257 & 0.024 & 0.012 & -0.016 & -0.042 & 0.89 & $\mathrm{H}$ \\
\hline 0.862318 & -0.2003 & -0.5661 & 0.038 & 0.020 & -0.005 & 0.021 & 0.84 & $\mathrm{~K}$ \\
\hline 0.862315 & -0.2058 & -0.5815 & 0.026 & 0.013 & -0.012 & 0.006 & 0.85 & $\mathrm{~L}$ \\
\hline 0.862355 & -0.2082 & -0.5885 & 0.013 & 0.006 & 0.004 & -0.007 & 0.86 & $\mathrm{P}$ \\
\hline 0.862314 & -0.2073 & -0.5857 & 0.020 & 0.010 & -0.014 & 0.002 & 0.86 & $\mathrm{Y}$ \\
\hline
\end{tabular}


Acknowledgements. This work was supported by the Russian Foundation for Basic Research, project no. 06-02-16966-a.

\section{References}

Aksnes, K., \& Franklin, F. 1976, AJ, 35, 513

Aksnes, K., Franklin, F., Millis, R., et al. 1984, AJ, 89, 280

Arlot, J.-E. 1984, A\&A, 138, 113

Arlot, J.-E., \& Thuillot, W. 1992, A\&AS, 92, 151

Descamps, P. 1992, Étude des effets de surface sur la réduction astrométrique des observations de phénomènes des satellites galiléens de Jupiter. Thèse de Doctorat. 1992, L'Observatoire de Paris

Descamps, P., Arlot, J.-E., Thuillot, W., et al. 1992, Icarus, 100, 235

Domingue, D., \& Verbiscer, A. 1997, Icarus, 128, 49

Emel'yanov, N. V. 1995, Astron. Rep., 39, 539

Emel'yanov, N. V. 1999, Sol. Sys. Res., 33, 133
Emel'yanov, N. V. 2000, Sol. Sys. Res., 34, 226

Emel'yanov, N. V. 2003, Sol. Sys. Res., 37, 314

Emel'yanov, N. V., \& Vashkov'yak, S. N. 2001, Sol. Sys. Res., 35, 513

Emelianov, N. V., Irsmambetova, T. R., Kiseleva, T. P., et al. 1999, A\&AS, 139, 47

Emelianov, N. V., Berejnoi, A. A., Vashkovjak, S. N, et al. 2000, A\&AS, 141, 433

Franklin, F. A., Africano, J., Allen, W., et al. 1991, AJ, 102, 806

Hapke, B. 1981, J. Geophys. Res., 1981, 86, 3039

Kaas, A. A., Aksnes, K., Franklin, F., \& Lieske J. 1999, AJ, 117, 1933

Lainey, V., Arlot, J. E., \& Vienne, A. 2004, A\&A, 427, 371

Makarova, E. A., Kharitonov, A. V., Kazachevskaya, T. V., et al. 1998, Baltic Astronomy, 7, 467

McEwen, A. S., Johnson, T. V., \& Matson, D. L. 1988, Icarus, 75, 450

Morrison, D., \& Morrison, N. D. 1977, Photometry of the Gallilean satellites, in Planetary satellites (Tucson: University of Arizona Press), 363

Vasundhara, R. 1994, A\&A, 281, 565

Vasundhara, R., Arlot, J.-E., Lainey, V., \& Thuillot, W. 1994, A\&A, 410, 337 